Research Article

\title{
Synchronized Survey Scan Approach Allows for Efficient Discrimination of Isomeric and Isobaric Compounds during LC-MS/MS Analyses
}

\author{
Keabetswe Masike and Ntakadzeni Madala \\ Department of Biochemistry, University of Johannesburg, P.O. Box 524, Auckland Park 2006, South Africa \\ Correspondence should be addressed to Ntakadzeni Madala; emadala@uj.ac.za
}

Received 23 January 2018; Accepted 28 February 2018; Published 1 April 2018

Academic Editor: Josep Esteve-Romero

Copyright (c) 2018 Keabetswe Masike and Ntakadzeni Madala. This is an open access article distributed under the Creative Commons Attribution License, which permits unrestricted use, distribution, and reproduction in any medium, provided the original work is properly cited.

\begin{abstract}
Liquid chromatography-mass spectrometry- (LC-MS-) based multiple reaction monitoring (MRM) methods have been used to detect and quantify metabolites for years. These approaches rely on the monitoring of various fragmentation pathways of multiple precursors and the subsequent corresponding product ions. However, MRM methods are incapable of confidently discriminating between isomeric and isobaric molecules and, as such, the development of methods capable of overcoming this challenge has become imperative. Due to increasing scanning rates of recent MS instruments, it is now possible to operate MS instruments both in the static and dynamic modes. One such method is known as synchronized survey scan (SSS), which is capable of acquiring a product ion scan (PIS) during MRM analysis. The current study shows, for the first time, the use of SSS-based PIS approach as a feasible identification feature of MRM. To achieve the above, five positional isomers of dicaffeoylquinic acids (diCQAs) were studied with the aid of SSS-based PIS method. Here, the MRM transitions were automatically optimized using a 3,5-diCQA isomer by monitoring fragmentation transitions common to all five isomers. Using the mixture of these isomers, fragmentation spectra of the five isomers achieved with SSS-based PIS were used to identify each isomer based on previously published hierarchical fragmentation keys. The optimized method was also used to detect and distinguish between diCQA components found in Bidens pilosa and their isobaric counterparts found in Moringa oleifera plants. Thus, the method was shown to distinguish (by differences in fragmentation patterns) between diCQA and their isobars, caffeoylquinic acid (CQA) glycosides. In conclusion, SSS allowed the detection and discrimination of isomeric and isobaric compounds in a single chromatographic run by producing a PIS spectrum, triggered in the automatic MS/MS synchronized survey scan mode.
\end{abstract}

\section{Introduction}

Plants produce a myriad of organic compounds referred to as secondary metabolites (natural products) which differ in their structure and biosynthetic origins. These metabolites undergo chemical modifications, such as conjugation $[1,2]$, and isomerization (positional and geometrical) [3-6], which further contribute to the high complexity of the plant metabolome. For instance, secondary metabolites such as hydroxycinnamic acid (HCA) derivatives have the potential to form conjugates with organic acids such as isocitric acid [1], tartaric acid [2, 7-9], and quinic acid [4, 10, 11], thus forming hydroxycinnamoyl-isocitric acid [1], hydroxycinnamoyl-tartaric acid [2, 7-9], and hydroxycinnamoyl-quinic acid $[4,10,11]$, respectively. The most common HCA derivatives include caffeic acid, ferulic acid, and $p$-coumaric acid, to name a few.

In addition, these HCA derivatives undergo isomerization to produce positional isomers such as di-acylated hydroxycinnamoyl-quinic acid derivatives like 1,3-dicaffeoylquinic acid (1,3-diCQA) or 1,5-diCQA [3-6]. As such, some HCA conjugates have been found to result in isobaric compounds which produce similar mass spectrometry (MS) fragmentation patterns [1], and this renders identification challenging from an analytical perspective. For instance, diCQA positional isomers, apart from being isobaric constituents of 
each other, also produce similar fragmentation patterns to structurally related molecules such as caffeoylquinic acid (CQA) glycosides [12-14], making identification in different plant species very challenging.

From the above, it can be surmised that secondary metabolites are diverse and, in some cases, are unique to specific plant species and, as such, can be used as chemotaxonomic markers [15-18]. Therefore, the unambiguous detection and identification of these metabolites using analytical techniques such as liquid chromatography-mass spectrometry (LC-MS) is of paramount importance. For targeted analysis, multiple reaction monitoring (MRM) using a triple quadrupole LC-MS/MS system can be employed to selectively screen for and detect and quantify metabolites of interest [19-22]. However, studies dedicated to providing specific fragmentation patterns that discriminate between isomeric and isobaric secondary metabolites during MRM analyses are limited [23, 24]. As such, a novel method/approach referred to as synchronized survey scan (SSS) is proposed as a possible way of discriminating structurally similar metabolites during MRM analyses, especially when they produce similar fragmentation transitions. Using a SSS function, we have demonstrated that positional isomers of diCQAs and their isobaric compounds, CQA glycosides, can be distinguished in a single chromatographic run. Here, authentic standards and plant extracts of Bidens pilosa [25] and Moringa oleifera were employed, since these plant species are reported to, respectively, accumulate/produce these compounds. Thus, the overall aim of the current study was to use the SSS approach as an orthogonal identification component of the MRM method for efficient discrimination of structurally related plant metabolites.

\section{Materials and Methods}

2.1. Materials. Authentic standards (with the purity of above 99.6\%) of dicaffeoylquinic acids (1,3-diCQA, 1,5-diCQA, 3,4-diCQA, 3,5-diCQA, and 4,5-diCQA) were purchased from Phytolab (Vestenbergsgreuth, Germany). Mass spectrometry grade $(99.9 \%)$ methanol was purchased from Romil Pure Chemistry (Cambridge, UK). Mass spectrometry grade formic acid (with the purity of above 96\%) was obtained from Sigma-Aldrich (St. Louis, MO, USA). The analytical column used was a reverse-phase Raptor biphenyl $(2.1 \times 100 \mathrm{~mm}, 3 \mu \mathrm{m})$ column purchased from Restek (Bellefonte, PA, USA).

\subsection{Methods}

2.2.1. Sample Preparation. A $1 \mathrm{mg} / \mathrm{mL}$ solution for each diCQA positional isomer was prepared with $100 \%$ methanol. The solution (for each positional isomer) was diluted $10 \times$ with $100 \%$ methanol. Furthermore, equal amounts (e.g., $40 \mu \mathrm{L}$ ) were taken from each positional isomer sample to prepare a mixed sample (e.g., of a final volume of $200 \mu \mathrm{L}$ ). The samples (individuals and the mixture) were placed in amber vials and subjected to HPLC-PDA analyses.
2.2.2. Metabolite Extraction. The dried leaves of B. pilosa and $M$. oleifera were pulverized, respectively, using a clean and dry quartz mortar and pestle. Extraction was conducted using an organic solvent-based extraction. The respective amounts of samples $(0.2 \mathrm{~g})$ were mixed with $2 \mathrm{~mL}$ of $50 \%$ aqueous methanol, and these extracts were placed (with the lids of the tubes closed to avoid evaporation) in a heating block at $60^{\circ} \mathrm{C}$ for $2 \mathrm{~h}$. The samples were sonicated for $30 \mathrm{~min}$ using an ultrasonic bath and then centrifuged at $9740 \times g$ for $10 \mathrm{~min}$ at $4^{\circ} \mathrm{C}$. The resulting supernatants were subjected to UHPLC-MS analyses.

\subsubsection{Ultra-High Performance Liquid Chromatography Tan-} dem Mass Spectrometry (UHPLC-MS/MS) Analysis. Once prepared, samples were analyzed on a Shimadzu Nexera 8050 UHPLC (Kyoto, Japan) fitted with a Raptor biphenyl analytical column, with the column temperature set at $40^{\circ} \mathrm{C}$. A binary solvent mixture consisting of MilliQ water made up of $0.1 \%$ formic acid (eluent A) and methanol made up of $0.1 \%$ formic acid (eluent B) at a constant flow rate of $0.4 \mathrm{~mL} / \mathrm{min}$ was used to analyze $2 \mu \mathrm{L}$ of the injected samples. For the gradient elution, the following conditions were used: isocratic $5 \%$ eluent $\mathrm{B}$ from $0 \mathrm{~min}$ to $1 \mathrm{~min}$, linear $5 \%-20 \%$ eluent B from $1 \mathrm{~min}$ to $5 \mathrm{~min}$, linear 20\%-90\% eluent B from $5 \mathrm{~min}$ to $40 \mathrm{~min}$, and isocratic $90 \%$ eluent B from $40 \mathrm{~min}$ to $45 \mathrm{~min}$. At the end of analysis, conditions were changed to the initial conditions (5\% eluent B) from $45 \mathrm{~min}$ to $48 \mathrm{~min}$ and finally, the column was reequilibrated with isocratic 5\% eluent $\mathrm{B}$ from $48 \mathrm{~min}$ to $52 \mathrm{~min}$. The data were acquired using a UV detector set at $325 \mathrm{~nm}$ and $330 \mathrm{~nm}$.

For the MS analysis, the chromatographic effluent was introduced to a MS source and ionized by electrospray (ESI). ESI conditions were as follows: the interface voltage was set at $3.0 \mathrm{kV}$ (in the negative ESI mode), the source temperature was $300^{\circ} \mathrm{C}$, nitrogen was used as the drying gas at the flow rate of $10.00 \mathrm{~L} / \mathrm{min}$, and as a nebulizing gas at a flow rate of $3.00 \mathrm{~L} / \mathrm{min}$. Argon was used as a collision gas with a pressure of $\pm 230 \mathrm{kPa}$ in the collision cell. Sensitive and qualitative analysis of isomeric and isobaric plant metabolites was achieved by developing a MRM and MRM-dependent product ion scan (PIS) method. The MRM transitions were developed or optimized using 3,5-diCQA as the sample of choice based on the work done by Clifford et al. [3]. According to Clifford and colleagues, 3,5-diCQA contains product ions (e.g., $m / z 353$ representing a caffeoylquinic acid moiety, $m / z 179$ representing a caffeic acid moiety, and $m / z$ 191 representing a quinic acid moiety) characteristic of all diCQA isomers, as well as CQA glycosides [13, 14]. The MRM transition parameters were automatically optimized to produce the transitions shown in Table 1. The dwell time for all the MRM transitions was $30 \mathrm{~ms}$.

A synchronized survey scan (SSS) function was selected which automatically performed MS/MS analysis when the precursor threshold peak intensity exceeded 2,000,000. This resulted in a combined MRM and a MRM-dependent PIS, both of which were produced in a single analysis. For the PIS mode, ions were collected at a mass range 100-1000 Da with a continuous scan time of $1 \mathrm{sec}$, at a collision energy of $25 \mathrm{eV}$. 
TABLE 1: MRM transitions automatically optimized using 3,5-diCQA.

\begin{tabular}{ccc}
\hline Precursor $(\mathrm{m} / z)$ & Transitions $(\mathrm{m} / z)$ & Collision energy $(\mathrm{eV})$ \\
\hline 515 & 353 & 18 \\
515 & 191 & 40 \\
515 & 179 & 28 \\
\hline
\end{tabular}

\section{Results and Discussion}

3.1. LC-MS/MS Method Optimization. In this study, LC-MS analysis was used to sensitively and qualitatively analyze isomeric and isobaric plant metabolites by developing a MRM-dependent product ion scan (PIS) method. Samples (authentic standards and plant samples) containing positional isomers of dicaffeoylquinic acids (diCQAs) and caffeoylquinic acid (CQA) glycosides were analyzed under reverse-phase chromatographic conditions using a Raptor biphenyl column with methanol as part of the binary solvent mixture. To identify the respective diCQA positional isomers, a sample made up of a mixture of the positional isomers (authentic standards) was analyzed, and it produced a chromatogram showing well-resolved peaks representing the five isomers (Figure 1; Table 2). The retention times (Rts) of the respective peaks were compared with the Rts of the individual (nonmixed) authentic standards, and the elution order under the abovementioned conditions was noted as 1,3-diCQA (Figure 1, A), 3,4-diCQA (Figure 1, B), 3,5-diCQA (Figure 1, C), 1,5-diCQA (Figure 1, D), and 4,5-diCQA (Figure 1, E). Furthermore, the respective product ion scan (PIS) spectra, triggered in the automatic MS/MS synchronized survey scan mode, were also referred to for the analysis of the fragmentation patterns of the diCQA positional isomers for further identification (Figure 2). Although the MRM transition parameters were automatically optimized using 3,5-diCQA, all five diCQA isomers were detected, since these compounds have been shown to share similar product ions $[3,11]$.

\subsection{MRM and MRM-Dependent Product Ion Scan Analysis of} Plant Extracts. After optimization, Bidens pilosa and Moringa oleifera plant extracts were analyzed under the abovementioned conditions (Section 3.1). When the MRM transition parameters, which were automatically optimized using 3,5-diCQA, were used to analyze extracts of $B$. pilosa and $M$. oleifera plant samples, several peaks were detected. Briefly, three peaks were detected in B. pilosa, and only two peaks were detected in $M$. oleifera. When compared, the two peaks detected from $M$. oleifera samples showed an earlier elution profile $(\mathrm{Rt}=4.89$ and 7.32$)$ and were thus observed to be more hydrophilic than the three peaks detected in B. pilosa samples, which showed a later elution profile $(\mathrm{Rt}=21.24$, 22.05, and 24.46). These observations are summarized in Table 2. Such chromatographic observations are important in scenarios whereby the analyzed plant sample contains both diCQAs and CQA glycosides, thus circumstances whereby the plant extract contains both the isomeric and isobaric compounds $[11,13,14,26]$.
The Rts of the peaks ( $\mathrm{Rt}=21.29,22.05$, and 24.61) found in B. pilosa samples were compared with those observed with the diCQA authentic standard samples, and the respective peaks were identified as 3,4-diCQA (Figure 3(a)), 3,5-diCQA (Figure 3(b)), and 4,5-diCQA (Figure 3(c)) (Figure 3; Table 2), since the fragmentation pattern of these peaks was identical to the fragmentation pattern observed in Figures 2(b), 2(c), and 2(e), respectively. For the peak representing 3,4-diCQA, the observed fragment ions were (Figure 3(a)) at $\mathrm{m} / \mathrm{z} 353$ ([caffeoylquinic acid- $\mathrm{H}]^{-}$) due to the loss of a caffeic acid moiety [3], at $m / z 335$ ([caffeoylquinic acid- $\left.\mathrm{H}_{2} \mathrm{O}-\mathrm{H}\right]^{-}$) due to the dehydration of the caffeoylquinic acid moiety which subsequently results in a lactone group on the quinic acid [27], at $m / z 191$ ([quinic acid-H] $]^{-}$) as a result of a loss of a caffeic acid moiety from a caffeoylquinic acid group, at $m / z$ 179 ([caffeic acid-H $]^{-}$) ascribed to the loss of the caffeoylquinic acid moiety (or the loss of a quinic acid moiety from a caffeoylquinic acid group), at $m / z 173$ ([quinic acid- $\mathrm{H}_{2} \mathrm{O}$ $\mathrm{H}]^{-}$) (base peak, bp) due to the dehydration of a quinic acid moiety, at $\mathrm{m} / \mathrm{z} 161$ ([caffeoylquinic acid-quinic acid moiety$\left.\mathrm{H}_{2} \mathrm{O}-2 \mathrm{H}\right]^{-}$) as a result of a loss of a dehydrated quinic acid moiety from a caffeoylquinic acid lactone, and at $\mathrm{m} / z 135$ ([caffeic acid- $\left.\mathrm{CO}_{2}-\mathrm{H}\right]^{-}$) due to the decarboxylation of a caffeic acid moiety (Figures 2(b) and 3(a); Table 2). The peak representing 3,5-diCQA showed fragment ions (Figure 3(b)) at $\mathrm{m} / z 353$ ([caffeoylquinic acid-H] $^{-}$), 191 ([quinic acid-H] $^{-}$) (bp), 179 ([caffeic acid-H $\left.]^{-}\right)$, and 135 ([caffeic acid-CO $\left.\left.{ }_{2}-\mathrm{H}\right]^{-}\right)$. Lastly, the peak identified as 4,5-diCQA showed product ions (Figure 3(c)) at $m / z 353$ ([caffeoylquinic acid-H] $]^{-}$) (bp), 191 ([quinic acid- $\left.\mathrm{H}]^{-}\right), 179$ ([caffeic acid- $\left.\mathrm{H}\right]^{-}$), and 173 ([quinic acid- $\left.\left.\mathrm{H}_{2} \mathrm{O}-\mathrm{H}\right]^{-}\right)$. It is worth noting that in the absence of authentic standards, the ion at $m / z 173$ is diagnostic of HCA derivatives acylated at position 4 on the quinic acid (e.g., 3,4-diCQA and 4,5-diCQA), as it has been noted in the work done by Clifford et al. $[3,10]$. However, to distinguish between 3,4-diCQA and 4,5 -diCQA an ion at $m / z 335$ is noteworthy, as its presence signifies the formation of a lactone group between a carboxylic group at position 1 and a hydroxyl group at position 5 on the quinic acid [27]. Thus, position 5 on the quinic acid need not be acylated to allow the formation of the lactone group as shown elsewhere [27]. Therefore, 3,4-diCQA and 4,5-diCQA can be distinguished based on the presence of the ion at $m / z 335$ on the $\mathrm{MS}^{2}$ spectra representing 3,4-diCQA [10].

The two peaks ( $\mathrm{Rt}=4.87$ and 7.30$)$ detected in the $M$. oleifera plant extracts were tentatively characterized as 3-caffeoylquinic acid (CQA) glycoside and 4-CQA glycoside (Table 2) [26]. The peak $(\mathrm{Rt}=4.87)$ representing 3-CQA glycoside showed fragment ions (Figure 3(d)) at $m / z 353$ ([caffeoylquinic acid- $\mathrm{H}]^{-}$) due to the neutral loss of a glycoside residue

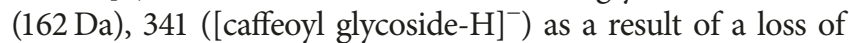
a quinic acid moiety, 191 ([quinic acid-H $]^{-}$) ascribed to the loss of a caffeoyl glycoside moiety, and 179 ([caffeic acid-H] $]^{-}$) (bp) due to the loss of the quinic acid and glycosyl moieties from the caffeoylquinic acid and caffeoyl glycoside groups, respectively. The peak $(\mathrm{Rt}=7.30)$ representing 4-CQA glycoside showed product ions (Figure 3(e)) at $m / z 353$ ([caffeoylquinic acid$\mathrm{H}]^{-}$), at $m / z 341$ ([caffeoyl glycoside- $\left.\mathrm{H}\right]^{-}$), at $m / z 191$ ([quinic acid-H $]^{-}$), at $m / z 179$ ([caffeic acid-H] $]^{-}$), and ion at $m / z 173$ ([quinic acid $\left.-\mathrm{H}_{2} \mathrm{O}-\mathrm{H}\right]^{-}$) (bp) due to the dehydration of 


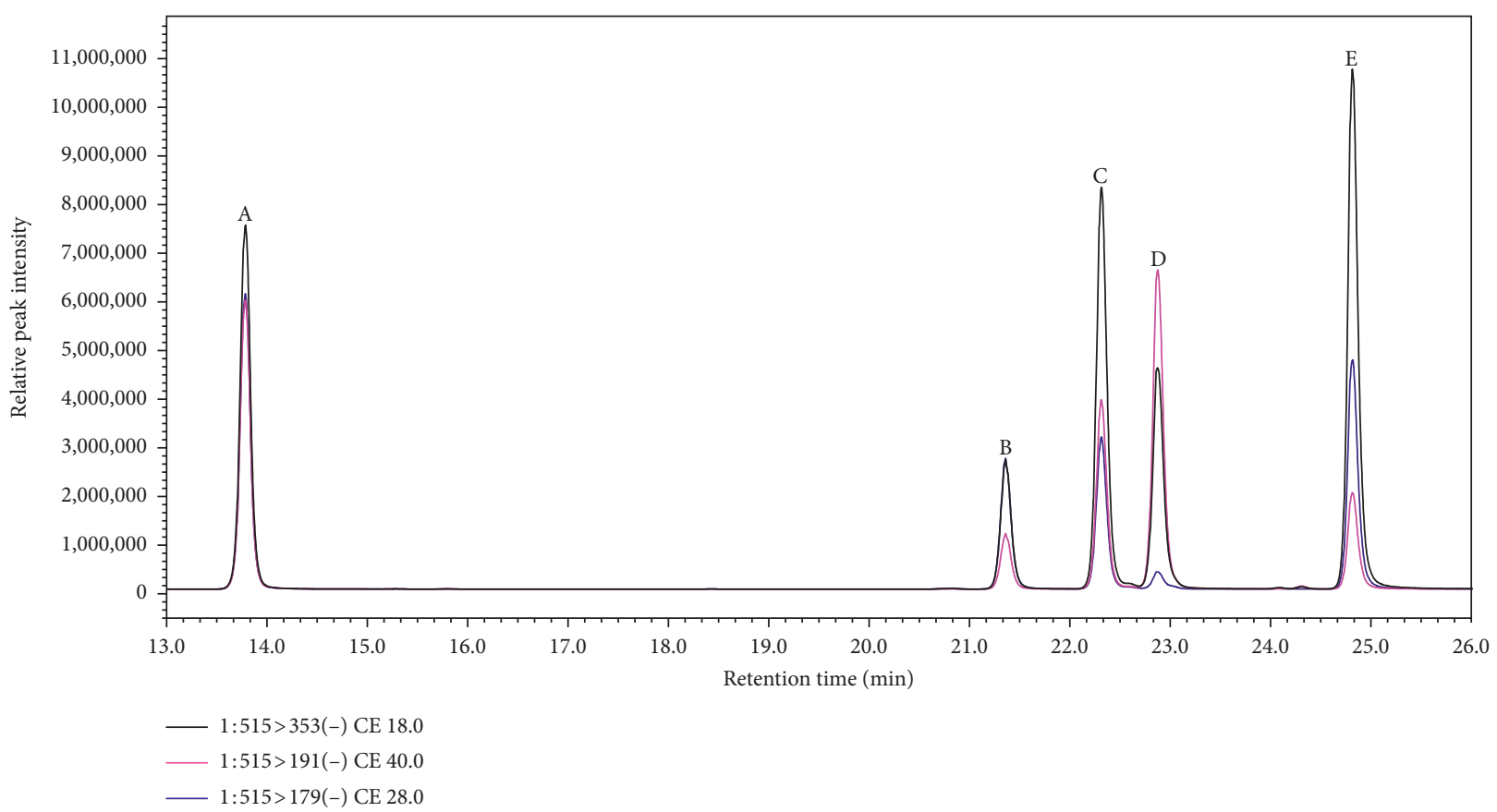

FIgURE 1: Chromatogram showing differences in the relative abundance of the MRM transitions of a sample containing a mixture of dicaffoylquinic acids (diCQAs) authentic standards: $\mathbf{A}=1,3$-diCQA, $\mathbf{B}=3,4$-diCQA, $\mathbf{C}=3,5$-diCQA, $\mathbf{D}=1,5$-diCQA, and $\mathbf{E}=4,5$-diCQA.

TABLE 2: MRM transitions and MRM-dependent product ion scan of isomeric and isobaric compounds from different sample types.

\begin{tabular}{|c|c|c|c|c|c|c|}
\hline $\begin{array}{l}\text { Sample } \\
\text { type }\end{array}$ & Compound & $\begin{array}{l}\text { Retention time } \\
(\mathrm{Rt})(\mathrm{min})\end{array}$ & $\begin{array}{l}\text { Precursor } \\
\quad(\mathrm{m} / z)\end{array}$ & $\begin{array}{c}\text { Optimal } \\
\text { transitions }\end{array}$ & $\begin{array}{c}\text { Collision } \\
\text { energy }(\mathrm{eV})\end{array}$ & MRM-dependent product ion \\
\hline \multirow{5}{*}{ Standard } & 1,3-O-dicaffeoylquinic acid & 13.71 & 515 & $\begin{array}{c}515>353 \\
515>179 / 191\end{array}$ & $\begin{array}{c}18 \\
28 / 40\end{array}$ & $\begin{array}{c}515 \rightarrow 353,335,191(b p), 179, \\
161,135\end{array}$ \\
\hline & 3,4-O-dicaffeoylquinic acid & 21.34 & 515 & $\begin{array}{c}515>179 / 353 \\
515>191\end{array}$ & $\begin{array}{c}28 / 18 \\
40\end{array}$ & $\begin{array}{c}515 \rightarrow 353,335,191,179,173 \\
(\mathrm{bp}), 161,135\end{array}$ \\
\hline & 3,5-O-dicaffeoylquinic acid & 22.25 & 515 & $\begin{array}{c}515>353 \\
515>191 / 179\end{array}$ & $\begin{array}{c}18 \\
40 / 28\end{array}$ & $515 \rightarrow 353,191(b p), 179,135$ \\
\hline & 1,5-O-dicaffeoylquinic acid & 22.82 & 515 & $\begin{array}{l}515>191 \\
515>353\end{array}$ & $\begin{array}{l}40 \\
18\end{array}$ & $515 \rightarrow 353,191(\mathrm{bp})$ \\
\hline & 4,5-O-dicaffeoylquinic acid & 24.75 & 515 & $\begin{array}{l}515>353 \\
515>179\end{array}$ & $\begin{array}{l}18 \\
28\end{array}$ & $\begin{array}{c}515 \rightarrow 353(\mathrm{bp}), 191,179,173, \\
135\end{array}$ \\
\hline \multirow{3}{*}{$\begin{array}{l}\text { Bidens } \\
\text { pilosa }\end{array}$} & 3,4-O-dicaffeoylquinic acid & 21.24 & 515 & $\begin{array}{c}515>179 / 353 \\
515>191\end{array}$ & $\begin{array}{c}28 / 18 \\
40\end{array}$ & $\begin{array}{c}515 \rightarrow 353,335,191,179,173 \\
(\mathrm{bp}), 161,135\end{array}$ \\
\hline & 3,5-O-dicaffeoylquinic acid & 22.05 & 515 & $\begin{array}{c}515>353 \\
515>191 / 179\end{array}$ & $\begin{array}{c}18 \\
40 / 28\end{array}$ & $515 \rightarrow 353,191(\mathrm{bp}), 179,135$ \\
\hline & 4,5-O-dicaffeoylquinic acid & 24.61 & 515 & $\begin{array}{l}515>353 \\
515>179\end{array}$ & $\begin{array}{l}18 \\
28\end{array}$ & $\begin{array}{c}515 \rightarrow 353(\mathrm{bp}), 191,179,173, \\
135\end{array}$ \\
\hline \multirow{2}{*}{$\begin{array}{l}\text { Moringa } \\
\text { oleifera }\end{array}$} & $\begin{array}{c}\text { 3-O-(4'-O-caffeoyl } \\
\text { glucosyl) quinic acid }\end{array}$ & 4.89 & 515 & $\begin{array}{c}515>179 \\
-\end{array}$ & 28 & $515 \rightarrow 353,341,191,179(\mathrm{bp})$ \\
\hline & $\begin{array}{l}\text { 4-O-(4'-O-caffeoyl } \\
\text { glucosyl) quinic acid }\end{array}$ & 7.32 & 515 & $\begin{array}{l}515>179 \\
515>353\end{array}$ & $\begin{array}{l}28 \\
18\end{array}$ & $\begin{array}{c}515 \rightarrow 353,341,191,179,173 \\
(\mathrm{bp})\end{array}$ \\
\hline
\end{tabular}

a quinic acid moiety. The presence and abundance of the peak at $m / z 173$ allowed characterization of the peak with the Rt of 7.30 as 4-CQA glycoside [10]. For both the peaks, the presence of the ion at $\mathrm{m} / \mathrm{z} 353$ and 341 suggests that the caffeoyl moiety forms an ester bond with the quinic acid (to produce an ion at $\mathrm{m} / z$ 353) and an ether bond with the glucose group (to produce an ion at $m / z$ 341). However, where the glycosyl group is connected on the caffeic acid catechol group $\left(\mathrm{C}-3^{\prime}\right.$ or $\left.\mathrm{C}-4^{\prime}\right)$ is not clear. According to Jaiswal et al., an intense ion (bp) at $m / z 323$ ([caffeoyl glycoside- $\left.\mathrm{H}_{2} \mathrm{O}-\mathrm{H}\right]^{-}$), due to the dehydration of a caffeoyl glycoside moiety, on the $\mathrm{MS}^{2}$ spectra, suggests connectivity at C- $3^{\prime}$ and an intense ion (bp) at $m / z 353$ on the $\mathrm{MS}^{2}$ spectra is characteristic of connectivity at C-4 $4^{\prime}[13,14]$. In this study, both ions were secondary ions and not base peaks, and the intensity of both peaks could have been influenced by the differences in the MS conditions/parameters [28]. Thus, due to the low abundance/absence of the ion at $m / z 323$ on the $\mathrm{MS}^{2}$ spectra (Figures 3(d) and 3(e)), the CQA glycosides were 


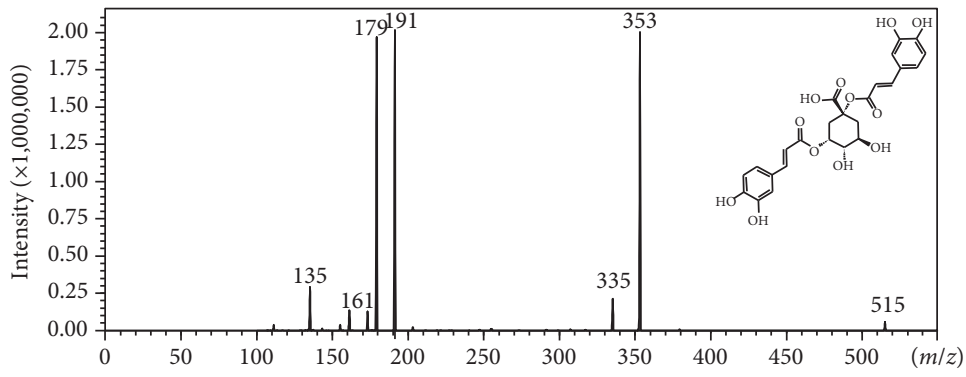

(a)

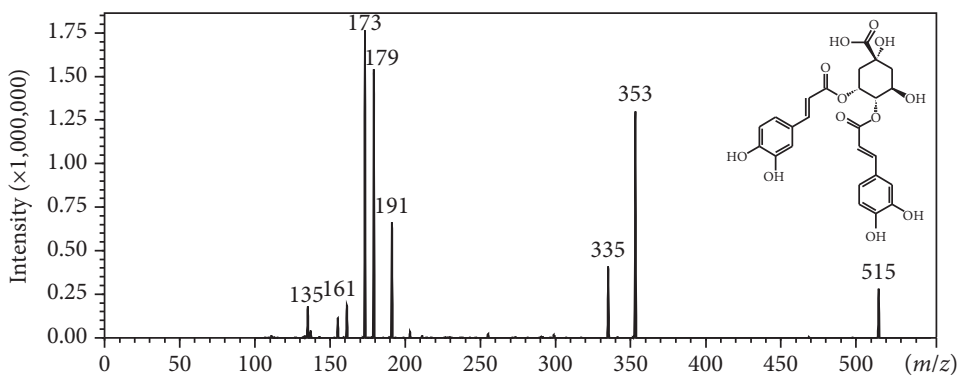

(b)

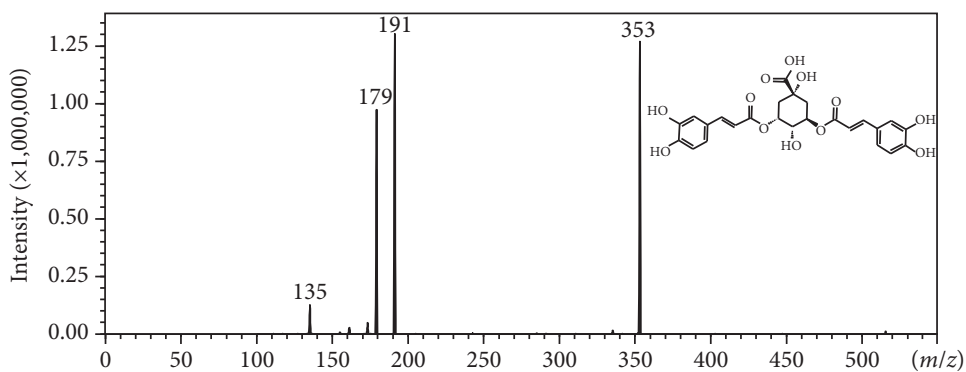

(c)

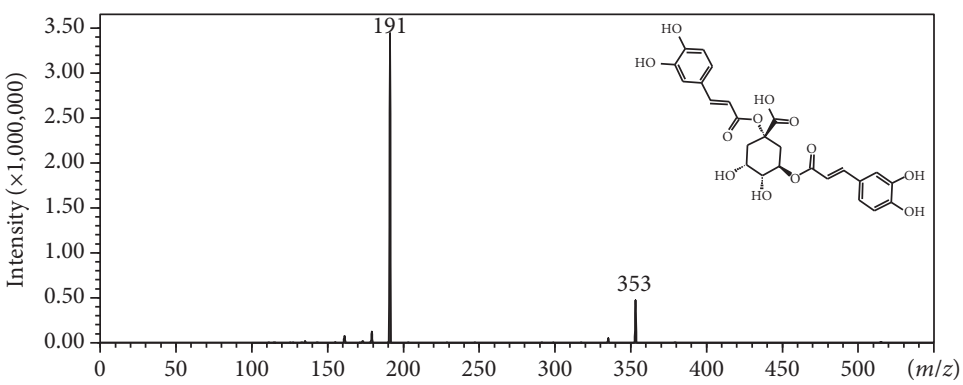

(d)

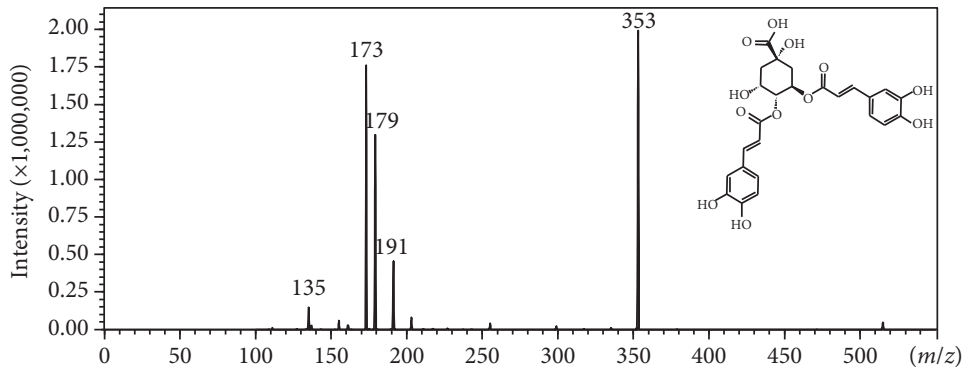

(e)

FIgURe 2: Product ion scan (PIS) spectra and fragmentation pathways of (a) 1,3-diCQA, (b) 3,4-diCQA, (c) 3,5-diCQA, (d) 1,5-diCQA, and (e) 4,5-diCQA authentic standards. 


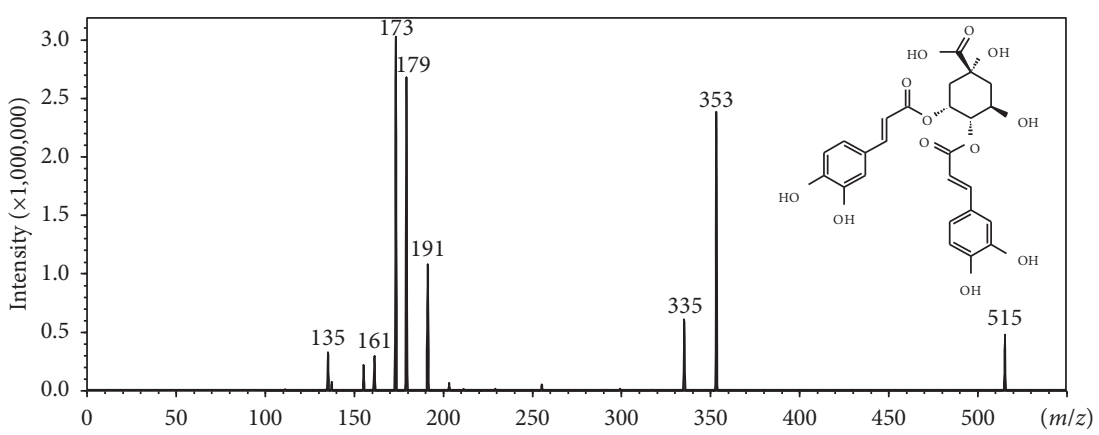

(a)

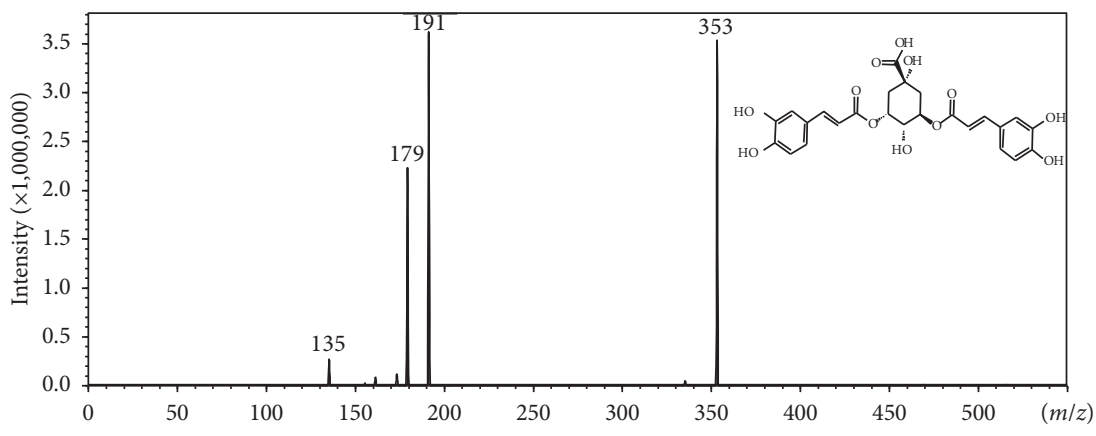

(b)

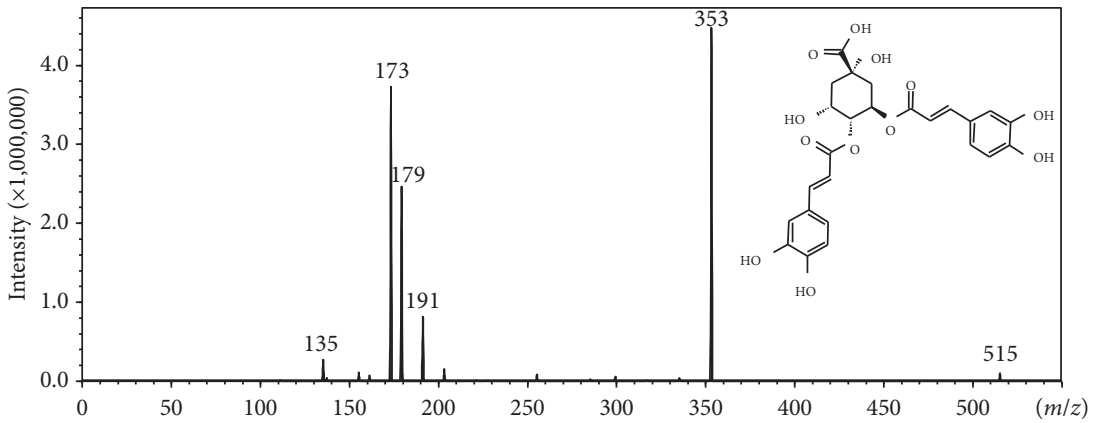

(c)

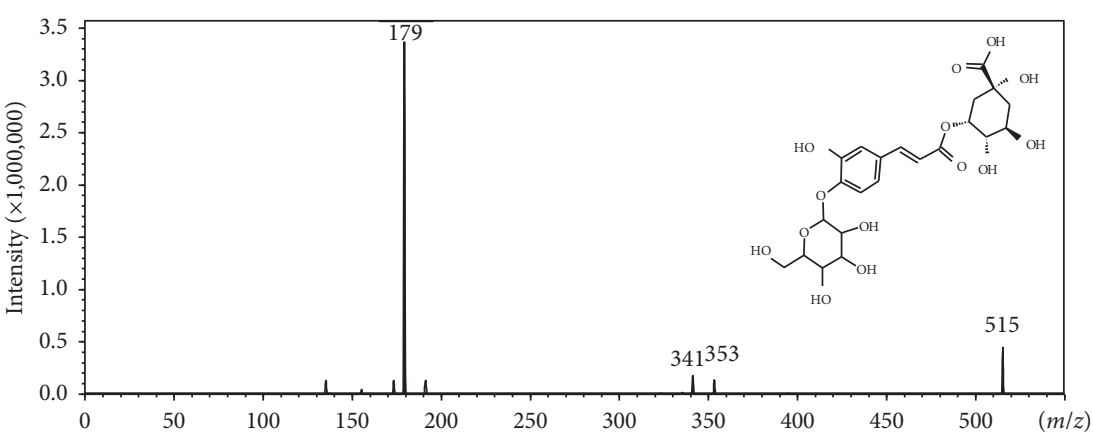

(d)

Figure 3: Continued. 


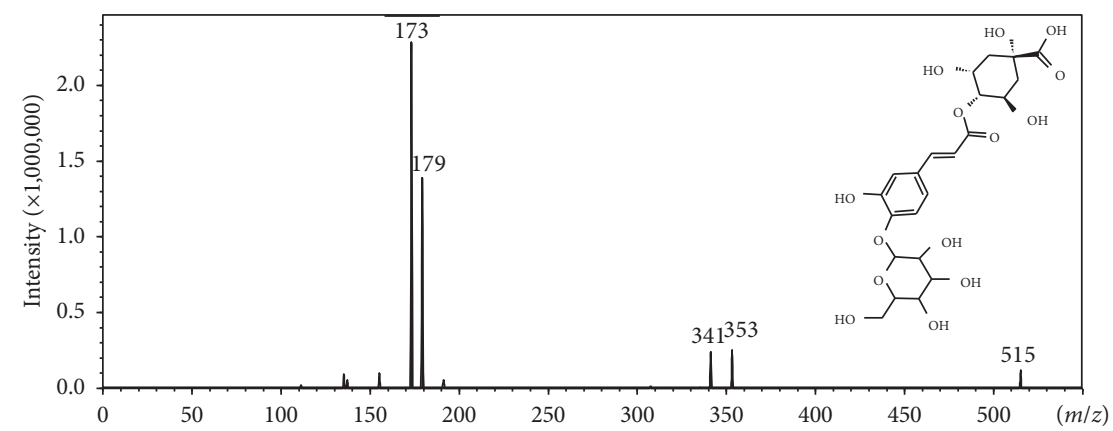

(e)

FIGURE 3: Product ion spectra and fragmentation pathway of diCQA positional isomers: (a) 3,4-diCQA, (b) 3,5-diCQA, and (c) 4,5-diCQA detected in Bidens pilosa plant extracts. Product ion spectra and fragmentation pathway of CQA glycoside positional isomers: (d) 3-O-(4'-Ocaffeoyl glucosyl) quinic acid and (e) 4-O-(4'-O-caffeoyl glucosyl) quinic acid detected in Moringa oleifera plant extracts.

putatively annotated as 3-O-(4'-O-caffeoyl glucosyl) quinic acid and 4-O-(4'-O-caffeoyl glucosyl) quinic acid (Figures 3(d) and 3(e); Table 2).

3.3. Synchronized Survey Scan (SSS). From the above, it is apparent that in the absence of the triggered product ion scan (PIS), the optimized MRM method would have detected the diCQA and the CQA glycosides as these compounds share similar transitions. However, the method would fail to differentiate between the structurally similar compounds and thus lead to misidentification. Thus, the novel approach, SSS, allowed the detection and differentiation of all five diCQA positional isomers (Figure 2) as well as two CQA glycoside positional isomers (Figures 3(d) and 3 (e)) in a single chromatographic analysis. Furthermore, the method allowed the discrimination of isobaric compounds such as diCQA and CQA glycosides. Here, an ion at $m / z 341$ was found to be present on the PIS MS/MS spectra of CQA glycosides. This ion represents caffeoyl glycoside [11] and, as seen from our results, it was only observed on the PIS spectra but not the MRM spectra. This is an indication that SSS dependent PIS allows other diagnostic ions to be used for the differentiation of closely related molecules, which otherwise would be impossible if only MRM transitions are relied upon. Thus, SSS produces an automatically performed MS/MS analysis which allows the simultaneous identification of these isomeric and isobaric compounds, without any ambiguity.

\section{Conclusion}

For the first time, we have successfully demonstrated synchronized survey scan (SSS) to be an efficient approach to detect and discriminate isomeric and isobaric plant metabolites. Briefly, this approach allowed the discrimination and identification of (1) diCQA positional isomers, (2) CQA glycosides positional isomers, and (3) isobaric compounds, diCQAs and CQA glycosides, in a single chromatographic run, albeit the developed MRM transition parameters were automatically optimized using the positional isomer 3,5-diCQA. Our results show that this method can be further applied in any method where isomers are expected. Furthermore, in phytochemistry, where identification is the key, SSS is expected to add a novel orthogonal feature during identification.

\section{Data Availability}

The data used to support the findings of this study are available from the corresponding author upon request.

\section{Conflicts of Interest}

The authors declare that they have no conflicts of interest.

\section{Acknowledgments}

The authors would like to thank the University of Johannesburg and the NRF for the financial support. The authors would also like to thank RESTEK for the RASP grant used to purchase the chromatographic columns. Dr. Riaan Meyer, Dr. Charles Yates, and Mr. Darryl Harris from Shimadzu, South Africa, are thanked for their technical assistance.

\section{References}

[1] K. Masike, M. I. Mhlongo, S. P. Mudau et al., "Highlighting mass spectrometric fragmentation differences and similarities between hydroxycinnamoyl-quinic acids and hydroxycinnamoyl-isocitric acids," Chemistry Central Journal, vol. 11, no. 29, pp. 1-7, 2017.

[2] K. Masike, F. Tugizimana, N. Ndlovu et al., "Deciphering the influence of column chemistry and mass spectrometry settings for the analyses of geometrical isomers of L-chicoric acid," Journal of Chromatography B, vol. 1052, pp. 73-81, 2017.

[3] M. N. Clifford, S. Knight, and N. Kuhnert, "Discriminating between the six isomers of dicaffeoylquinic acid by LC-MS $n$," Journal of Agricultural and Food Chemistry, vol. 53, no. 10, pp. 3821-3832, 2005.

[4] M. N. Clifford, J. Kirkpatrick, N. Kuhnert, H. Roozendaal, and P. R. Salgado, "LC-MS $n$ analysis of the cis isomers of chlorogenic acids," Food Chemistry, vol. 106, no. 1, pp. 379385, 2008.

[5] M. M. Makola, P. Steenkamp, I. A. Dubery, M. M. Kabanda, and N. E. Madala, "Preferential alkali metal adduct formation 
by cis geometrical isomers of dicaffeoylquinic acids allows for efficient discrimination from their trans isomers during ultrahigh-performance liquid chromatography/quadrupole timeof-fight mass spectrometry," Rapid Communications in Mass Spectrometry, vol. 30, no. 8, pp. 1011-1018, 2016.

[6] K. Masike, I. Dubery, P. Steenkamp, E. Smit, and E. Madala, "Revising reverse-phase chromatographic behavior for efficient differentiation of both positional and geometrical isomers of dicaffeoylquinic acids," Journal of Analytical Methods in Chemistry, vol. 2018, Article ID 8694579, 11 pages, 2018.

[7] J. Lee and C. F. Scagel, "Chicoric acid: chemistry, distribution, and production," Frontiers in Chemistry, vol. 1, no. 40, pp. 1-17, 2013.

[8] J. Lee and C. F. Scagel, "Chicoric acid levels in commercial basil (Ocimum basilicum) and Echinacea purpurea products," Journal of Functional Foods, vol. 2, no. 1, pp. 77-84, 2010.

[9] B. Khoza, S. Gbashi, P. Steenkamp, P. Njobeh, and N. Madala, "Identification of hydroxylcinnamoyl tartaric acid esters in Bidens pilosa by UPLC-tandem mass spectrometry," South African Journal of Botany, vol. 103, pp. 95-100, 2016.

[10] M. N. Clifford, K. L. Johnston, S. Knight, and N. Kuhnert, "Hierarchical scheme for LC-MS ${ }^{n}$ identification of chlorogenic acids," Journal of Agricultural and Food Chemistry, vol. 51, no. 10, pp. 2900-2911, 2003.

[11] E. N. Ncube, M. I. Mhlongo, L. A. Piater, P. A. Steenkamp, I. A. Dubery, and N. E. Madala, "Analyses of chlorogenic acids and related cinnamic acid derivatives from Nicotiana tabacum tissues with the aid of UPLC-QTOF-MS/MS based on the insource collision-induced dissociation method," Chemistry Central Journal, vol. 8, no. 66, pp. 1-10, 2014.

[12] M. N. Clifford, W. Wu, J. Kirkpatrick, and N. Kuhnert, "Profiling the chlorogenic acids and other caffeic acid derivatives of herbal Chrysanthemum by LC-MS ${ }^{n}$," Journal of Agricultural and Food Chemistry, vol. 55, no. 3, pp. 929-936, 2007.

[13] R. Jaiswal, S. Deshpande, and N. Kuhnert, "Profiling the chlorogenic acids of Rudbeckia hirta, Helianthus tuberosus, Carlina acaulis and Symphyotrichum novae-angliae leaves by LC-MS ${ }^{n}$," Phytochemical Analysis, vol. 22, no. 5, pp. 432-441, 2011.

[14] R. Jaiswal, E. A. Halabi, M. G. E. Karar, and N. Kuhnert, "Identification and characterisation of the phenolics of Ilex glabra L. Gray (Aquifoliaceae) leaves by liquid chromatography tandem mass spectrometry," Phytochemistry, vol. 106, pp. 141-155, 2014.

[15] L. M. Calabria, V. P. Emerenciano, M. J. Ferreira, M. T. SCotti, and T. J. Mabry, "A phylogenetic analysis of tribes of the Asteraceae based on phytochemical data," Natural Product Communications, vol. 2, pp. 277-285, 2007.

[16] C. Makita, L. Chimuka, P. Steenkamp, E. Cukrowska, and E. Madala, "Comparative analyses of flavonoid content in Moringa oleifera and Moringa ovalifolia with the aid of UHPLC-qTOF-MS fingerprinting," South African Journal of Botany, vol. 105, pp. 116-122, 2016.

[17] M. E. P. Martucci, R. C. de Vos, C. A. Carollo, and L. GobboNeto, "Metabolomics as a potential chemotaxonomical tool: application in the genus Vernonia schreb," PloS One, vol. 9, no. 4, Article ID e93149, 2014.

[18] A. Vallverdú-Queralt, A. Medina-Remon, M. Martínez-Huélamo, O. Jáuregui, C. Andres-Lacueva, and R. M. Lamuela-Raventos, "Phenolic profile and hydrophilic antioxidant capacity as chemotaxonomic markers of tomato varieties," Journal of Agricultural and Food Chemistry, vol. 59, no. 8, pp. 3994-4001, 2011.

[19] O. Aizpurua-Olaizola, J. Omar, P. Navarro, M. Olivares, N. Etxebarria, and A. Usobiaga, "Identification and quantification of cannabinoids in Cannabis sativa L. plants by high performance liquid chromatography-mass spectrometry," Analytical and Bioanalytical Chemistry, vol. 406, no. 29, pp. 7549-7560, 2014.

[20] N. R. Kitteringham, R. E. Jenkins, C. S. Lane, V. L. Elliott, and B. K. Park, "Multiple reaction monitoring for quantitative biomarker analysis in proteomics and metabolomics," Journal of Chromatography B, vol. 877, no. 13, pp. 1229-1239, 2009.

[21] M. Martínez-Huélamo, S. Tulipani, X. Torrado, R. Estruch, and R. M. Lamuela-Raventós, "Validation of a new LCMS/MS method for the detection and quantification of phenolic metabolites from tomato sauce in biological samples," Journal of Agricultural and Food Chemistry, vol. 60, no. 11, pp. 4542-4549, 2012.

[22] X. Su, R. Lin, S. Wong, S. Tsui, and S. Kwan, "Identification and characterisation of the Chinese herb Langdu by LCMS/MS analysis," Phytochemical Analysis, vol. 14, no. 1, pp. 40-47, 2003.

[23] L. Abrankó and B. Szilvássy, "Mass spectrometric profiling of flavonoid glycoconjugates possessing isomeric aglycones," Journal of Mass Spectrometry, vol. 50, no. 1, pp. 71-80, 2015.

[24] M. E. Fridén and P. J. Sjöberg, "Strategies for differentiation of isobaric flavonoids using liquid chromatography coupled to electrospray ionization mass spectrometry," Journal of Mass Spectrometry, vol. 49, no. 7, pp. 646-663, 2014.

[25] S. Gbashi, P. Njobeh, P. Steenkamp, H. Tutu, and N. Madala, "The effect of temperature and methanol-water mixture on Pressurized Hot Water Extraction (PHWE) of anti-HIV analogous from Bidens pilosa," Chemistry Central Journal, vol. 10, no. 37, pp. 1-12, 2016.

[26] C. Makita, L. Chimuka, E. Cukrowska et al., "UPLC-qTOFMS profiling of pharmacologically important chlorogenic acids and associated glycosides in Moringa ovalifolia leaf extracts," South African Journal of Botany, vol. 108, pp. 193-199, 2017.

[27] L. Dawidowicz and R. Typek, "Transformation of chlorogenic acids during the coffee beans roasting process," European Food Research and Technology, vol. 243, no. 3, pp. 379-390, 2016.

[28] N. E. Madala, P. A. Steenkamp, L. A. Piater, and I. A. Dubery, "Collision energy alteration during mass spectrometric acquisition is essential to ensure unbiased metabolomic analysis," Analytical and Bioanalytical Chemistry, vol. 404, no. 2, pp. 367-372, 2012. 

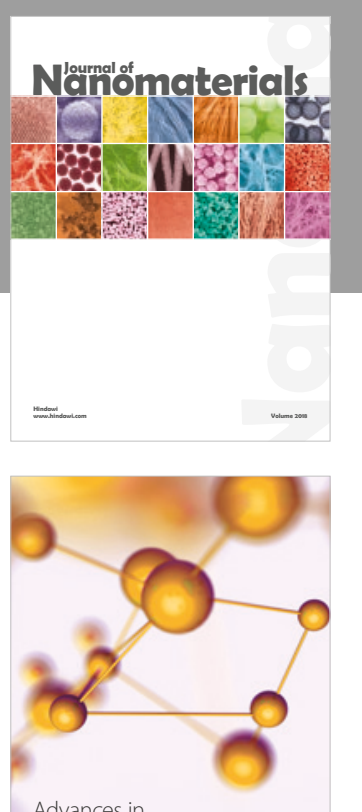

Physical Chemistry
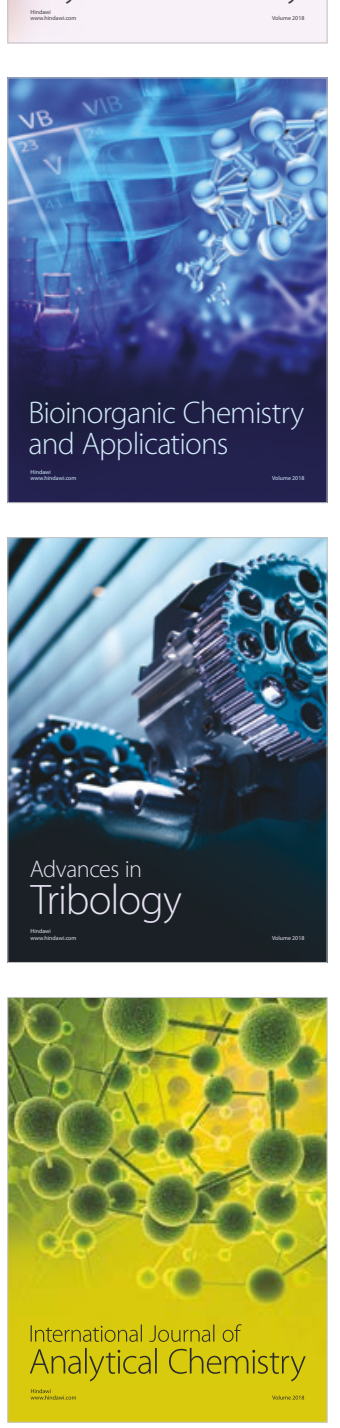

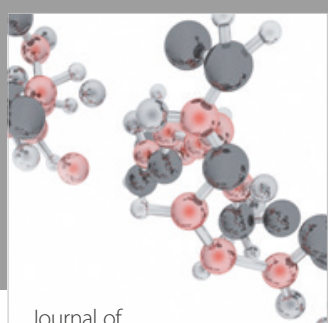

Analytical Methods

in Chemistry

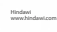

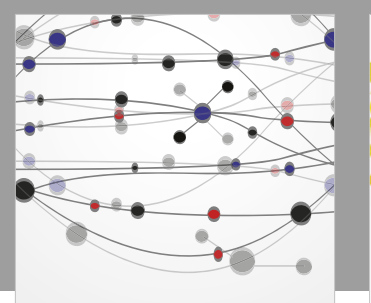

The Scientific World Journal

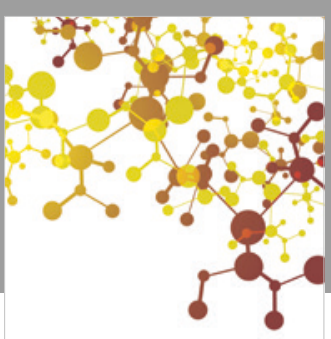

Journal of

Applied Chemistry
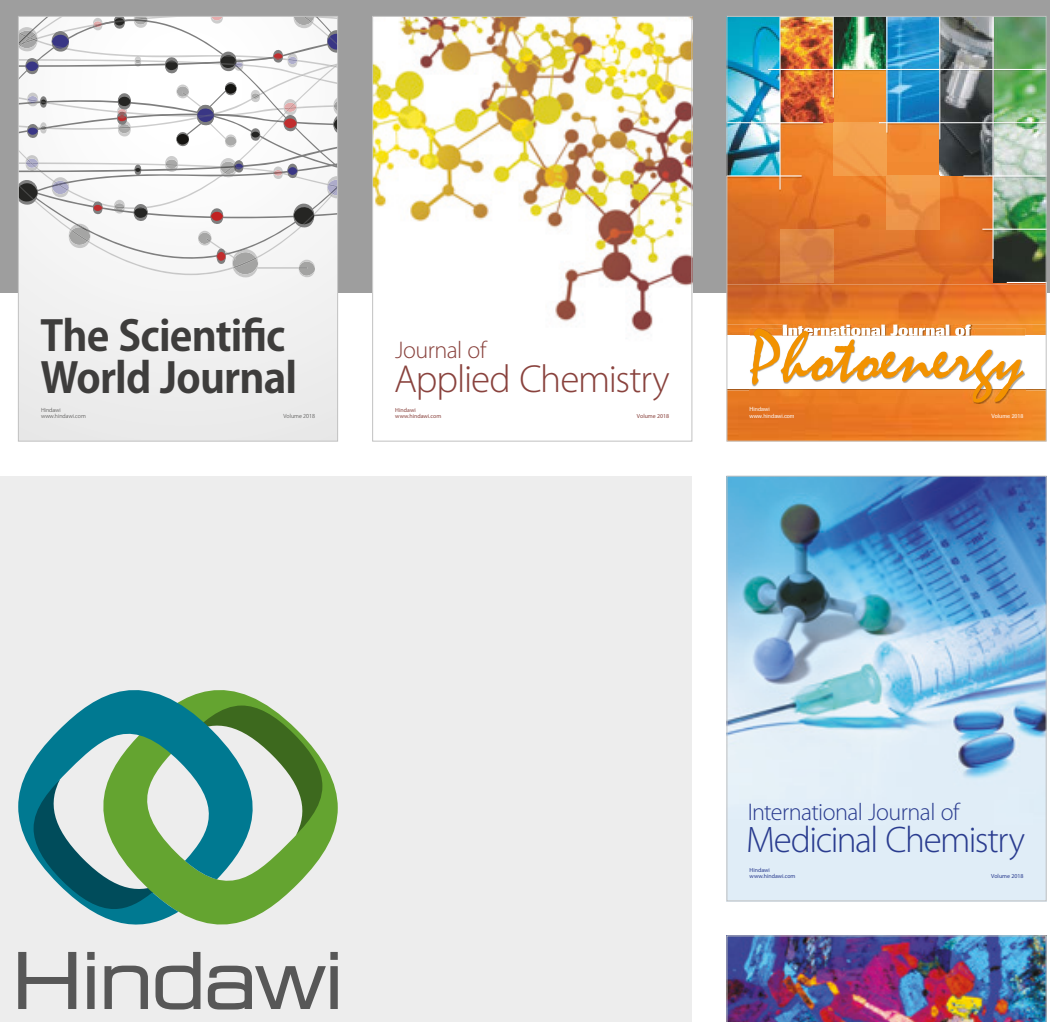

Submit your manuscripts at

www.hindawi.com
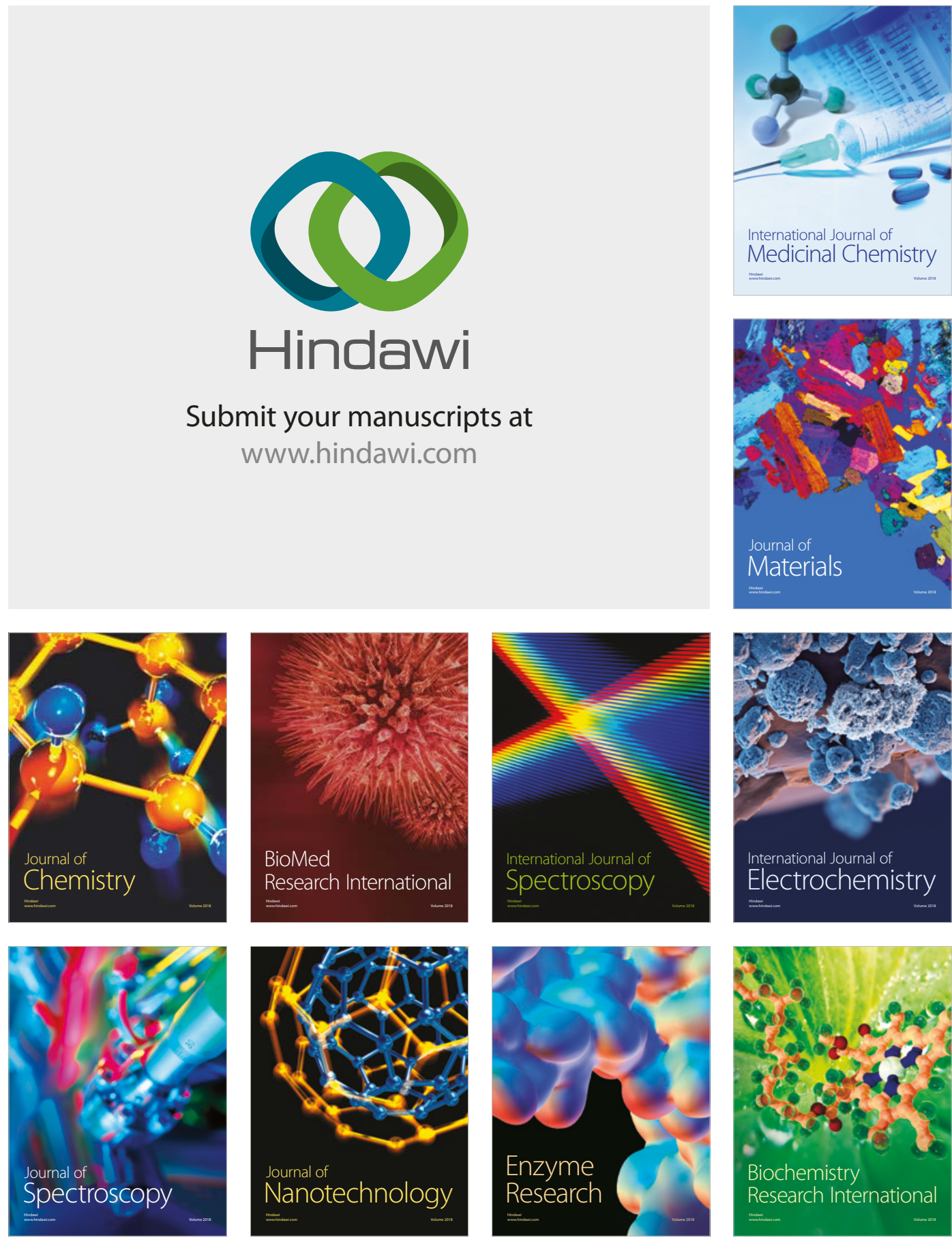
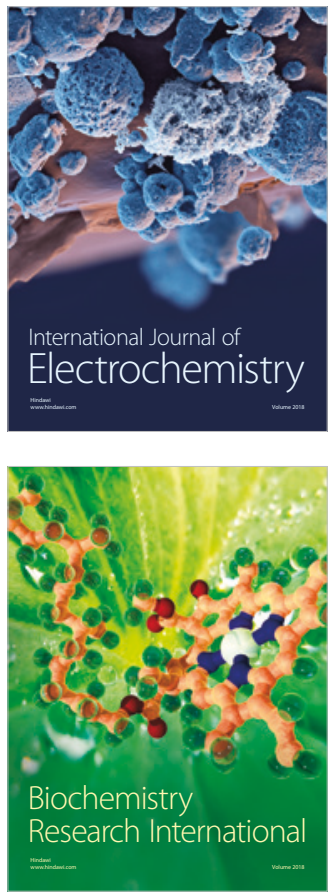